\title{
Newly diagnosed diabetes mellitus as a risk factor for serious liver disease
}

\author{
Liane Porepa MD, Joel G. Ray MD MSc, Paula Sanchez-Romeu MMath, \\ Gillian L. Booth MD MSc
}

Previously published at www.cmaj.ca

\section{ABSTRACT}

Background: The negative impact of diabetes mellitus is well recognized, yet little is known about the effect of this disease on the liver, an organ susceptible to nonalcoholic fatty liver disease related to insulin resistance. We evaluated whether adults with newly diagnosed diabetes were at increased risk of serious liver disease.

Methods: We used administrative health databases for the province of Ontario (1994-2006) to perform a populationbased matched retrospective cohort study. The exposed group comprised 438069 adults with newly diagnosed diabetes. The unexposed comparison group - those without known diabetes - consisted of 2059708 individuals, matched 5:1 to exposed persons, by birth year, sex and local health region. We excluded individuals with preexisting liver or alcohol-related disease. The primary study outcome was the subsequent development of serious liver disease, namely, liver cirrhosis, liver failure and its sequelae, or receipt of a liver transplant.

Results: The incidence rate of serious liver disease was 8.19 per 10000 person-years among those with newly diagnosed diabetes and 4.17 per 10000 person-years among those without diabetes. The unadjusted hazard ratio was 1.92 (95\% confidence interval $[\mathrm{Cl}] 1.83-2.01)$. After adjustment for age, income, urban residence, health care utilization and pre-existing hypertension, dyslipidemia, obesity and cardiovascular disease, the hazard ratio was 1.77 (95\% Cl 1.68-1.86).

Interpretation: Adults with newly diagnosed diabetes appeared to be at higher risk of advanced liver disease, also known as diabetic hepatopathy. Whether this reflects nonalcoholic fatty liver disease or direct glycemic injury of the liver remains to be determined.

$\mathrm{P}$ arallel to adult obesity, ${ }^{1}$ the prevalence of type 2 diabetes mellitus has risen to an unprecedented level over the past decade. ${ }^{2}$ The negative impact of diabetes on the retinal, renal, nervous and cardiovascular systems is well recognized, ${ }^{3,4}$ yet little is known about its effect on the liver. It has recently been realized that hepatic dysregulation in the setting of obesity is marked by oxidative stress and steatosis related to insulin resistance. ${ }^{5}$ The ensuing effect is nonalcoholic fatty liver disease, a common spectrum of disease ranging from simple steatosis (fatty infiltration) to inflammatory steatohepatitis to possible long-term injury (fibrosis and cirrhosis) and eventual liver failure. ${ }^{6}$ Recent evidence has even suggested that elevated levels of aminotransferase may be a marker of future risk of diabetes.?

Existing guidelines do not advocate screening for liverrelated complications among persons with diabetes, making the liver a potentially neglected target organ. Diabetes-associated hepatopathy has been described largely in uncontrolled patient series $;{ }^{8}$ however, those reports did not assess the development of serious liver disease. In one large cohort study based on computerized national databases of the US Department of Veterans Affairs, the incidence of chronic nonalcoholic liver disease was nearly two times higher among those with diabetes. ${ }^{9}$ However, the study sample consisted almost entirely of older men requiring care in hospital, for whom the duration of diabetes was unknown.

We conducted a population-based study to evaluate whether adults with newly diagnosed diabetes were at increased risk of serious liver disease relative to matched controls without diabetes.

\section{Methods}

We used administrative health claims for the province of Ontario to perform this population-based, matched retrospective cohort study. All of Ontario's approximately 12 million residents are registered in the universally available Ontario Health Insurance Plan, which covers most aspects of health care and for which there is no user fee.

\section{Participants}

We identified as the exposed group all adults aged 30 to 75 years with diabetes that was newly diagnosed between Apr. 1, 1994, and Mar. 31, 2006. Each exposed individual was matched to five randomly selected individuals without a diagnosis of diabetes by year of birth, sex and the geographic region in which they resided (known as Local

From the Department of Medicine (Porepa, Ray, Booth), University of Toronto, Toronto, Ont.; the Keenan Research Centre, Li Ka Shing Knowledge Institute of St. Michael's Hospital (Ray, Booth), Toronto, Ont.; and the Institute for Clinical Evaluative Sciences (Ray, Sanchez-Romeu, Booth), Toronto, Ont.

CMAJ 2010. DOI:10.1503/cmaj.092144 
Health Integration Networks). We excluded anyone with a diagnosis of liver disease, alcoholism or an alcohol-related medical condition in the 36-month period before the index date, on the basis of International Classification of Diseases codes listed in hospital records (9th revision codes before Apr. 1, 2002; 10th revision codes from Apr. 1, 2002, onward) or diagnostic codes listed in physicians' services claims (Appendix 1, available at www.cmaj.ca/cgi/content /full/cmaj.092144/DC1).

\section{Exposure}

Diabetes status was determined from the Ontario Diabetes Database, a provincial registry that uses administrative health claims from hospital admissions and ambulatory services to identify individuals with diagnosed diabetes. ${ }^{10}$ Briefly, people with one or more hospital admissions or two or more claims for physicians' services within a two-year period bearing a diagnosis of diabetes (International Classification of Diseases, 9th revision, code 250.X or International Classification of Diseases, 10th revision, codes E10 to E14) are included in the Ontario Diabetes Database. Once a case has been entered into the database, it remains there until a record appears of the person's death or migration out of province. This algorithm had a sensitivity of $86 \%$ and a specificity of $97 \%$ for identifying patients with documented diabetes when validated against primary care charts. ${ }^{10}$ Although the Ontario Diabetes Database does not discriminate between type 1 and type 2 diabetes, the likelihood of type 2 diabetes is very high among those in whom the disease is newly diagnosed after age 30 .

We defined the index date for an exposed individual as the date of his or her first record in the Ontario Diabetes Database. To rule out pre-existing diabetes, we included in the exposed group only those with a new record in the database after a minimum observation period of 36 months. We excluded from the unexposed group individuals who appeared to be free of diabetes at baseline but who entered the database at a later date.

\section{Outcomes and covariates}

The main study outcome was a composite of serious liver disease, specifically liver cirrhosis, liver failure or liver transplantation. We used hospital records to identify relevant codes from the International Classification of Diseases (9th or 10th revision) for each outcome (Appendix 1). For liver transplantation, we used codes from the Canadian Classification of Procedures (corresponding to years in which the 9th revision of the International Classification of Diseases was in effect) or the Canadian Classification of Interventions (corresponding to years in which the 10th revision of the International Classification of Diseases was in effect), in conjunction with claims submitted by physicians for these services. The secondary study outcomes were liver cirrhosis, liver failure and liver transplantation as separate outcomes. Some specific diagnoses associated with liver cirrhosis and liver failure included hepatic necrosis, hepatic coma, portal hypertension, hepatorenal syndrome and esophageal varices.
Covariates that we believed represented potential confounders were age, income quintile, and urban or rural residence, each at the index date; a diagnosis of arterial hypertension, dyslipidemia, obesity or cardiovascular disease within the 36 months before the index date (Appendix 1); number of hospital admissions within the 36 months before the index date; and number of primary care visits within the 12 months before the index date. The last of these was intended to account for potential differences in access to care and the likelihood of having had an assessment of liver function, for example. We assigned income quintiles on the basis of the median household income level of individuals' respective neighbourhoods of residence, according to the index date closest to the 1996, 2001 or 2006 Canadian Census, with " 1 " representing the lowest income quintile and " 5 " the highest income quintile. We defined cardiovascular disease as a prior admission to hospital for acute myocardial infarction or stroke, on the basis of codes from the International Classification of Diseases, 9th or 10th revision. ${ }^{4}$ Arterial hypertension was based on a validated algorithm that used diagnostic codes from hospital and physicians' service claims. ${ }^{11,12}$ Dyslipidemia and obesity were also based on physician service claims, but we have not validated these diagnoses. Physical measures (e.g., body mass index), information on medication use and ethnicity, and biochemical parameters (e.g., fasting blood glucose or alanine aminotransferase) were not available in the databases that we used.

We captured study outcomes and covariates from three provincial health care administrative databases that are linked deterministically to the Ontario Diabetes Database. We obtained hospital admission records from the Canadian Institute for Heath Information Discharge Abstract Database. The anonymized discharge abstracts contained the person's unique encrypted health care number, his or her age and sex, the date of admission and up to 25 diagnoses from the International Classification of Diseases, 9th or 10th revision. The database of the Ontario Hospital Insurance Plan contains records of all physicians' billing for outpatient and inpatient services, including a service date and a single diagnosis. The Registered Persons Database contains health care identifiers for all eligible individuals, as well as their dates of death, if applicable.

The health care databases were linked anonymously using encrypted individual health card numbers to safeguard patient confidentiality. The study was approved by the Research Ethics Board at Sunnybrook Health Sciences Centre.

\section{Statistical analysis}

The period of observation for members of the exposed group began on the index date for diagnosis of diabetes. For each member in the unexposed group, observation began on the same date as the index date of the exposed person to whom he or she was matched. We performed time-to-event analysis up to Mar. 31, 2008. We censored individuals who experienced a study outcome event; those in whom viral hepatitis, alcoholic liver disease, primary biliary cirrhosis or toxic liver failure were diagnosed (Appendix 1); those who died; and those who reached the end of the study period. 
We determined incidence rates of serious liver disease and its components for participants with and without diabetes. We derived crude and adjusted hazard ratios (HRs) and 95\% confidence intervals (CIs) from multivariable Cox proportional hazards models, with unexposed persons without diabetes serving as the reference group. Hazard ratios above 1.0 indicate a greater risk of serious liver disease among those with newly diagnosed diabetes. We further adjusted for age (continuous in years); income quintile; urban residence; hypertension, dyslipidemia, obesity, cardiovascular disease and number of hospital admissions in the 36 months before study entry; and number of primary care visits in the 12 months before study entry. We generated survival curves for serious liver disease from the unadjusted Cox model. We assessed the proportional hazards assumption using log-negative-log plots; the survival curves for those with and without diabetes did not cross, and generally remained parallel.

Using the same multivariable model, we further stratified the adjusted HRs for serious liver disease according to age (30-49, 50-64 and $\geq 65$ years), sex, rural versus urban residence and income quintile. With the exception of the age strata, we did not perform adjustments to the variables that were used for stratification.

We also evaluated incidence rates and adjusted HRs for serious liver disease in the presence of three pre-existing features of the metabolic syndrome within the 36 months before the index date, namely, hypertension, dyslipidemia and pre-existing obesity. The multivariable models were the same as described previously, but we did not perform adjustment on the feature of the metabolic syndrome that was being explored.

All $p$ values were two-sided, at a significance level of 0.05 .

\section{Results}

Our study sample consisted of 2497777 adults aged 30 to 75 years, of whom 438069 had newly diagnosed diabetes and were matched to 2059708 individ uals without diabetes (Table 1). The overall mean age was 55.3 (standard deviation 11.4) years, 1090067 of the participants (43.6\%) were women, and 2155167 $(86.3 \%)$ were living in an urban area. Preceding entry into the study, diagnosed hypertension, dyslipidemia and obesity were more prevalent among those with newly diagnosed diabetes, and these participants had more frequent visits to a primary care practitioner in the year preceding study entry (Table 1).
After a median duration of follow-up of 6.4 years, totalling 17176801 person-years, serious liver disease was identified in 2463 individuals with newly diagnosed diabetes and 5902 unaffected controls (Table 2, Appendix 2, available at www.cmaj.ca/cgi/content/full/cmaj.092144/DC1). The corresponding incidence rates were 8.19 per 10000 person-years for those with diabetes and 4.17 per 10000 person-years for controls (crude HR 1.92, 95\% CI 1.83-2.01) (Table 2). After adjustment for potential confounders, the HR for serious liver disease was 1.77 (95\% CI 1.68-1.86) (Table 2). The incidence rate and adjusted HR associated with diabetes were more pronounced for liver cirrhosis $(2.55,95 \%$ CI $2.35-2.76)$ than for liver transplantation $(1.31,95 \%$ CI 1.05-1.64), of which the latter accounted for about $7 \%$ of all cases of serious liver disease (Table 2).
Table 1: Characteristics of 2497777 participants in a study of serious liver disease in relation to presence of newly diagnosed diabetes mellitus

\begin{tabular}{|c|c|c|}
\hline \multirow[b]{2}{*}{ Characteristic } & \multicolumn{2}{|c|}{ Study group; no. (\%) of participants* } \\
\hline & $\begin{array}{l}\text { Newly diagnosed } \\
\text { diabetes mellitus } \\
n=438069\end{array}$ & $\begin{array}{c}\text { No diabetes mellitus } \\
n=2059708\end{array}$ \\
\hline Age, yr, mean (SD) & $55.5(11.4)$ & $55.3(11.4)$ \\
\hline \multicolumn{3}{|l|}{ Age group, yr } \\
\hline $30-40$ & $51034(11.6)$ & $245625(11.9)$ \\
\hline $41-50$ & $98623(22.5)$ & $473215(23.0)$ \\
\hline $51-60$ & $127140(29.0)$ & $601598(29.2)$ \\
\hline $61-70$ & $115732(26.4)$ & $528474(25.7)$ \\
\hline $71-75$ & $45540(10.4)$ & $210796(10.2)$ \\
\hline Sex, female & $188791(43.1)$ & $901276(43.8)$ \\
\hline \multicolumn{3}{|l|}{ Income quintile } \\
\hline Q1 (lowest) & 95760 (21.9) & $366670(17.8)$ \\
\hline Q2 & $94571(21.6)$ & $403749(19.6)$ \\
\hline Q3 & $88831(20.3)$ & $415133(20.2)$ \\
\hline Q4 & $83167(19.0)$ & $420538(20.4)$ \\
\hline Q5 (highest) & $73707(16.8)$ & $447573(21.7)$ \\
\hline Unknown & $2033 \quad(0.5)$ & $6045 \quad(0.3)$ \\
\hline Urban residence & $378152(86.3)$ & 1777015 (86.3) \\
\hline \multicolumn{3}{|l|}{ Medical history before study entry } \\
\hline Hypertension within 36 mo & $148303(33.9)$ & 352732 (17.1) \\
\hline Dyslipidemia within 36 mo & $93820(21.4)$ & $296652(14.4)$ \\
\hline Obesity within 36 mo & $48513(11.1)$ & 78730 \\
\hline $\begin{array}{l}\text { Cardiovascular disease within } \\
36 \text { mo }\end{array}$ & $11456 \quad(2.6)$ & $16951 \quad(0.8)$ \\
\hline $\begin{array}{l}\text { No. of hospital admissions within } \\
36 \text { mo, mean (SD) }\end{array}$ & $0.28(0.79)$ & $0.16(0.63)$ \\
\hline $\begin{array}{l}\text { No. of primary care visits within } \\
12 \text { mo, mean (SD) }\end{array}$ & 6.9 (7.3) & $4.2 \quad(6.6)$ \\
\hline \multicolumn{3}{|l|}{ Person-years of follow-up } \\
\hline Total no. & 3009109 & 14167692 \\
\hline Median (IQR) & $6.4(3.9-9.6)$ & $6.4(3.9-9.6)$ \\
\hline
\end{tabular}

$\mathrm{IQR}$ = interquartile range, $\mathrm{Q}=$ quintile, $\mathrm{SD}=$ standard deviation.

*Unless otherwise indicated. 
Table 2: Risk of serious liver disease in association with newly diagnosed diabetes mellitus in 2497777 study participants

\begin{tabular}{|c|c|c|c|c|}
\hline \multirow{2}{*}{ Study outcome } & \multicolumn{2}{|c|}{$\begin{array}{l}\text { Study group; no. (incidence rate } \\
\text { per } 10000 \text { person-years) }\end{array}$} & & \\
\hline & $\begin{array}{c}\text { Newly diagnosed } \\
\text { diabetes mellitus } \\
n=438069\end{array}$ & $\begin{array}{l}\text { No diabetes mellitus } \\
n=2059708\end{array}$ & \multicolumn{2}{|c|}{$\mathrm{HR}(95 \% \mathrm{Cl}) *$} \\
\hline Primary: any serious liver disease & $2463(8.19)$ & $5902(4.17)$ & $1.92(1.83-2.01)$ & $1.77(1.68-1.86)$ \\
\hline Liver failure and its sequelae & $1760(5.84)$ & 4485 (3.16) & $1.80(1.70-1.91)$ & $1.69(1.59-1.79)$ \\
\hline Liver transplantation & $174(0.58)$ & $449(0.32)$ & $1.80(1.51-2.15)$ & $1.31(1.05-1.64)$ \\
\hline
\end{tabular}

$\mathrm{Cl}=$ confidence interval, $\mathrm{HR}=$ hazard ratio.

*Comparison of those with newly diagnosed diabetes mellitus and matched individuals without diabetes mellitus.

†Adjusted for age (continuous, in years); income quintile; urban residence; presence of arterial hypertension, dyslipidemia, obesity or cardiovascular disease within 36 months before study entry; number of hospital admissions within 36 months before study entry; and number of primary care visits within 12 months before study entry.

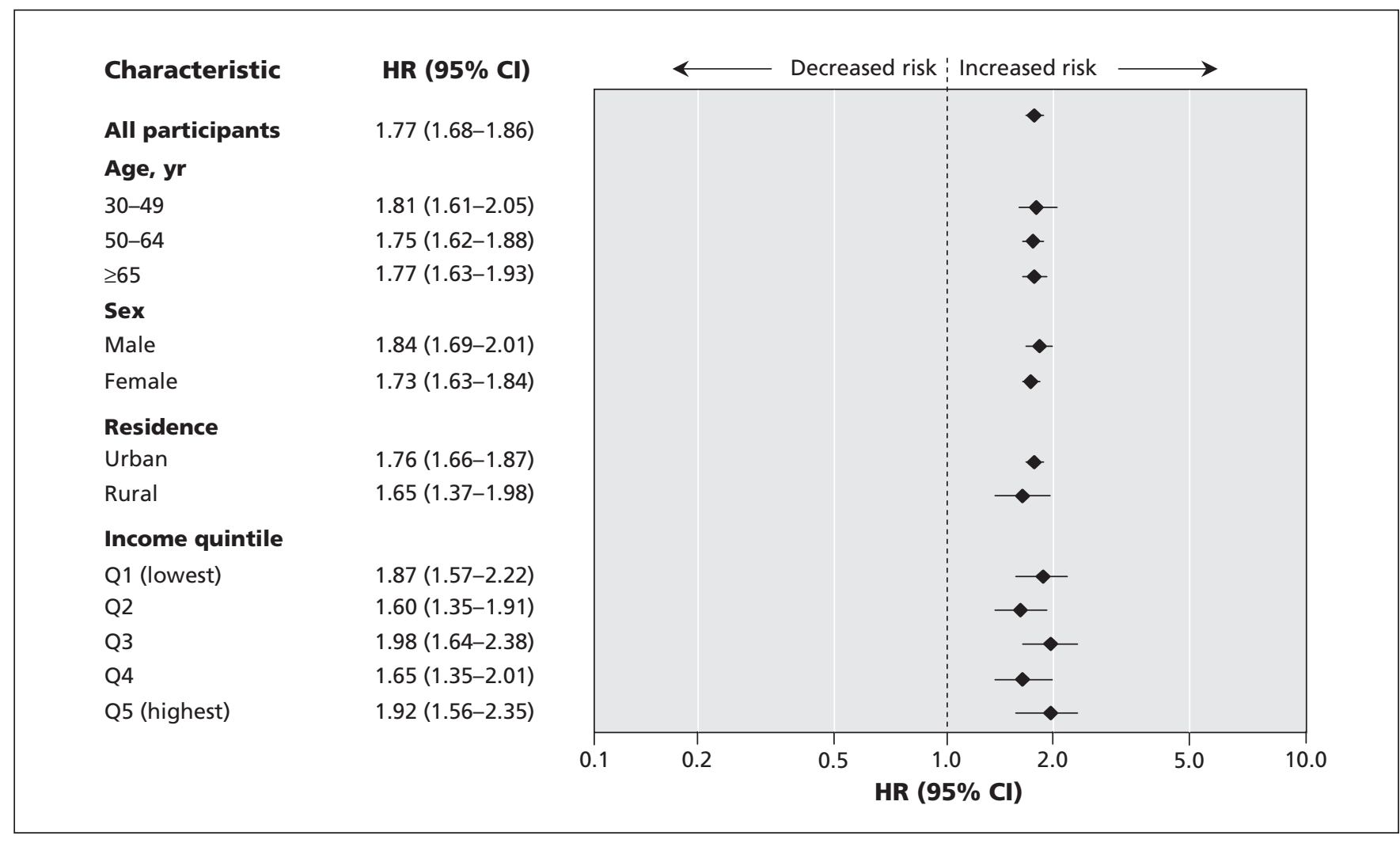

Figure 1: Risk of serious liver disease according to participants' characteristics at study entry, comparing those with newly diagnosed diabetes mellitus and matched individuals without diabetes mellitus. Hazard ratios (HRs) were adjusted for age (continuous, in years); income quintile; urban residence; presence of hypertension, dyslipidemia, obesity or cardiovascular disease within 36 months before study entry; number of hospital admissions within 36 months before study entry; and number of primary care visits within 12 months before study entry. With the exception of the age stratum, adjustment was not performed on the variable being stratified. $\mathrm{Cl}=\mathrm{confi}-$ dence interval.

The overall higher risk of serious liver disease in association with newly diagnosed diabetes was consistent across demographic strata at study entry (Figure 1). Individuals with newly diagnosed diabetes had a higher associated risk of serious liver disease relative to unaffected individuals who were free of diabetes or a pre-existing diagnostic feature of the metabolic syndrome (Table 3). In the absence of diabetes, those with pre-existing hypertension or obesity, but not dyslipidemia, were also at higher risk of serious liver disease, but less so than those with diabetes (Table 3). 
Table 3: Risk of serious liver disease in the presence of three pre-existing features of the metabolic syndrome - chronic hypertension, dyslipidemia and obesity — in relation to the presence of newly diagnosed diabetes mellitus in 2497777 participants

Risk of serious liver disease

Feature of metabolic syndrome $\leq 36 \mathrm{mo}$ before study entry and/or diabetes

\author{
No. (\%) of all participants \\ $n=2497777$ \\ Incidence rate per \\ 10000 person-years
}

Adjusted HR* $(95 \% \mathrm{Cl})$

\begin{tabular}{lccc}
\hline Hypertension & & & \\
Neither hypertension nor diabetes mellitus & $1706976(68.3)$ & 3.8 & 1.00 (reference) \\
\hline Hypertension without diabetes mellitus & $352732(14.1)$ & 6.3 & $1.23(1.14-1.31)$ \\
\hline Diabetes with or without hypertension & $438069(17.5)$ & 8.2 & $1.88(1.78-1.98)$ \\
Dyslipidemia & & 4.3 & 1.00 (reference) \\
Neither dyslipidemia nor diabetes mellitus & $1763056(70.6)$ & 3.5 & $0.68(0.62-0.74)$ \\
\hline Dyslipidemia without diabetes mellitus & $296652(11.9)$ & 8.2 & $1.66(1.57-1.75)$ \\
\hline Diabetes with or without dyslipidemia & $438069(17.5)$ & 4.1 & 1.00 (reference) \\
Obesity & & 4.9 & $1.16(1.01-1.33)$ \\
\hline Neither obesity nor diabetes mellitus & $1980773(79.3)$ & 8.2 & $1.78(1.70-1.88)$ \\
\hline Obesity without diabetes mellitus & $78935(3.2)$ & $438069(17.5)$ & \\
\hline Diabetes with or without obesity & & & \\
\hline
\end{tabular}

$\mathrm{Cl}=$ confidence interval, $\mathrm{HR}=$ hazard ratio

*Adjusted for age (continuous, in years); income quintile; urban residence; presence of arterial hypertension, dyslipidemia, obesity or cardiovascular disease within 36 months before study entry; number of hospital admissions within 36 months before study entry; and number of primary care visits within 12 months before study entry. Adjustment was not performed on the feature of the metabolic syndrome that was being explored.

\section{Interpretation}

In this study, adults with newly diagnosed diabetes had a significantly greater risk of serious liver disease than those without diabetes. This result held across demographic strata and with adjustment for other known risk factors for nonalcoholic fatty liver disease. Diabetes, with or without pre-existing hypertension, dyslipidemia or obesity, conferred a higher risk of serious liver disease than any of the three other conditions in isolation.

Our research complements a large cohort study conducted by the US Department of Veterans Affairs, which found a similarly higher risk of chronic nonalcoholic liver disease among veterans with diabetes who had been admitted to hospital. ${ }^{9}$ Although our findings and those of the US study edge forward the idea that diabetes may be harmful to the liver, the question remains of whether this effect extends beyond the metabolic syndrome. In the United Kingdom Prospective Diabetes Study, the metabolic syndrome was present in $61 \%$ of adults with diabetes, ${ }^{13}$ which indicates that it is not easy to entirely separate the effects of these related conditions. We found that obesity and arterial hypertension were each individually associated with a modestly increased risk of serious liver disease; however, the risk was highest in the presence of diabetes. In other studies, the combination of diabetes and the metabolic syndrome posed a higher risk for stroke ${ }^{14}$ and myocardial infarction ${ }^{15}$ than either condition alone. For a variety of reasons, the same phenomenon may be true for liver disease. Hepatic fat content increases in these individuals in parallel to insulin resistance and glucose dysregulation. ${ }^{16}$ The final evolution to type 2 diabetes is marked by $\beta$-cell failure, ${ }^{17}$ at which point hyperglycemia develops in parallel with increases in plasma triglycerides and the hepatic insulin resistance index, potentially further augmenting the fatty liver burden. ${ }^{18}$ Thus, we posit that the presence of overt diabetes reflects more severe insulin resistance, a greater fatty load in the liver ${ }^{19,20}$ and potentially worse hepatic inflammation and injury. ${ }^{21,22}$ At the same time, we do not know whether the hepatocyte is directly susceptible to glucotoxicity.

\section{Limitations}

Our study had several limitations. First, through our use of the Ontario Diabetes Database, some persons with diabetes might have been assigned to the unexposed group, despite our efforts to exclude them. Although we could not distinguish between newly diagnosed type 1 and type 2 diabetes, the age of diagnosis (30 to 75 years) would favour the far more prevalent form of type 2 diabetes in this age group. We also considered whether our observation might be temporally incorrect, meaning that diabetes developed as a complication of cirrhosis (so-called "hepatogenous diabetes"). ${ }^{23}$ However, hepatogenous diabetes typically does not arise until liver cirrhosis has reached an advanced stage, with overt clinical disease, ${ }^{23}$ and it may be reversible after liver transplantation. ${ }^{24}$ Moreover, in our study and the study by the US Department of Veterans Affairs, ${ }^{9}$ patients with diagnosed liver disease at baseline were excluded; as such, the results of these two studies suggest that diabetes preceded the onset of advanced-stage liver injury. However, we could not rule out subclinical pre-existing liver disease among participants before study entry. Similarly, we could not assess whether nonalcoholic fatty liver disease or steatohepatitis was the underlying cause of serious liver disease, because there are no codes for these conditions in 
the 9th or 10th revision of the International Classification of Diseases, and biochemical, radiological and histological data were not available in our data sources. Despite the exclusion of individuals with ethanol-related disorders and the censoring of those with viral hepatitis, our study sample likely retained some affected individuals.

Although the administrative databases that we used may accurately capture diabetes and arterial hypertension, ${ }^{10,11}$ the $11 \%$ prevalence of obesity among those with newly diabetes was lower than expected, ${ }^{25,26}$ which suggests that obesity was undercaptured in this population, partly because body mass index and waist circumference were not available. Finally, the ethnicity of study participants was not known.

\section{Conclusions}

Our observed incidence rate for serious liver disease of 8.19 per 10000 person-years among adults with newly diagnosed diabetes was lower than published rates for diabetes-related end-stage renal disease (20 to 30 per 10000 person-years) ${ }^{27}$ and sight-threatening diabetic retinopathy (45 per 10000 person-years).$^{28}$ Each of these conditions seriously affects quality of life. However, when the liver fails, there is no equivalent form of management, such as hemodialysis or retinal photocoagulation. Thus, although diabetic hepatopathy is potentially less common, it may be appropriate for addition to the list of target-organ conditions related to diabetes, such as glomerulopathy, retinopathy and neuropathy. Annual screening for liver disease might be accomplished by means of a simple biochemical analyte such as alanine aminotransferase. However, before screening can be considered, the efficacy of primary and secondary preventive measures, such as weight loss and glycemic and lipid control, must be validated by good evidence akin to that completed for adults and children with isolated fatty liver and no diabetes. ${ }^{29}$

This article has been peer reviewed.

\section{Competing interests: None declared.}

Contributors: Liane Porepa participated in study conception and design, drafting of the manuscript and approval of the final version. Joel Ray participated in study conception and design, analysis of the data, drafting and revision of the manuscript and approval of the final version. Paula SanchezRomeu participated in study design, analysis of the data, revision of the manuscript and approval of the final version. Gillian Booth participated in study conception and design, analysis of the data, drafting and revision of the manuscript and approval of the final version.

Funding: This work was funded by the Banting and Best Diabetes Centre at the University of Toronto. The study was also supported by the Institute for Clinical Evaluative Sciences (ICES), which is funded by an annual grant from the Ontario Ministry of Health and Long-Term Care (MOHLTC). The opinions, results and conclusions reported in this paper are those of the authors and are independent from those of the funding sources. No endorsement by ICES or the Ontario MOHLTC is intended or should be inferred.

\section{REFERENCES}

1. Mokdad AH, Ford ES, Bowman BA, et al. Prevalence of obesity, diabetes, and obesity-related health risk factors, 2001. JAMA 2003;289:76-9.

2. Lipscombe LL, Hux JE. Trend in diabetes prevalence, incidence, and mortality in Ontario, Canada (1995-2005): a population-based study. Lancet 2007;369:750-6.
3. Orasanu G, Plutzky J. The pathologic continuum of diabetic vascular disease. J Am Coll Cardiol 2009;53:S35-42.

4. Booth GL, Kapral MK, Fung K, et al. Relation between age and cardiovascular disease in men and women with diabetes compared with nondiabetic people: a population-based retrospective cohort study. Lancet 2006;368:29-36.

5. Youssef W, McCullough AJ. Diabetes mellitus, obesity, and hepatic steatosis. Semin Gastrointest Dis 2002;13:17-30.

6. Ong JP, Pitts A, Younossi ZM. Increased overall mortality and liver-related mortality in nonalcoholic fatty liver disease. J Hepatol 2008;49:608-12.

7. Goessling W, Massaro JM, Vasan RS, et al. Aminotransferase levels and 20-year risk of metabolic syndrome, diabetes, and cardiovascular disease. Gastroenterology 2008;135:1935-44.

8. Allison ME, Wreghitt T, Palmer CR, et al. Evidence for a link between hepatitis C virus infection and diabetes mellitus in a cirrhotic population. $J$ Hepatol 1994;21:1135-9.

9. El-Serag HB, Everhart JE. Diabetes increases the risk of acute hepatic failure. Gastroenterology 2002;122:1822-8.

10. Hux JE, Ivis F, Flintoft V, et al. Diabetes in Ontario: determination of prevalence and incidence using a validated administrative data algorithm. Diabetes Care 2002;25:512-6.

11. Tu K, Campbell NR, Chen ZL, et al. Accuracy of administrative databases in identifying patients with hypertension. Open Med 2007;1:e18-26.

12. Tu K, Chen Z, Lipscombe LL; Canadian Hypertension Education Program Outcomes Research Taskforce. Prevalence and incidence of hypertension from 1995 to 2005: a population-based study. CMAJ 2008;178:1429-35.

13. Cull CA, Jensen CC, Retnakaran R, et al. Impact of the metabolic syndrome on macrovascular and microvascular outcomes in type 2 diabetes mellitus: United Kingdom Prospective Diabetes Study 78. Circulation 2007;116:2119-26.

14. Najarian RM, Sullivan LM, Kannel WB, et al. Metabolic syndrome compared with type 2 diabetes mellitus as a risk factor for stroke: the Framingham Offspring Study. Arch Intern Med 2006;166:106-11.

15. Hanefeld M, Koehler C, Gallo S, et al. Impact of the individual components of the metabolic syndrome and their different combinations on the prevalence of atherosclerotic vascular disease in type 2 diabetes: the Diabetes in Germany (DIG) study. Cardiovasc Diabetol 2007;6:13.

16. Cali AM, De Oliveira AM, Kim H, et al. Glucose dysregulation and hepatic steatosis in obese adolescents: Is there a link? Hepatology 2009;49:1896-903.

17. Porte D Jr, Kahn SE. Beta-cell dysfunction and failure in type 2 diabetes: potential mechanisms. Diabetes 2001;50 Suppl 1:S160-3

18. Toledo FG, Sniderman AD, Kelley DE. Influence of hepatic steatosis (fatty liver) on severity and composition of dyslipidemia in type 2 diabetes. Diabetes Care 2006;29:1845-50.

19. Donnelly KL, Smith CI, Schwarzenberg SJ, et al. Sources of fatty acids stored in liver and secreted via lipoproteins in patients with nonalcoholic fatty liver disease. J Clin Invest 2005;115:1343-51.

20. Wasada T, Kasahara T, Wada J, et al. Hepatic steatosis rather than visceral adiposity is more closely associated with insulin resistance in the early stage of obesity. Metabolism 2008;57:980-5.

21. Liangpunsakul S, Chalasani N. Unexplained elevations in alanine aminotransferase in individuals with the metabolic syndrome: results from the third National Health and Nutrition Survey (NHANES III). Am J Med Sci 2005;329:111-6.

22. Solga SF, Clark JM, Alkhuraishi AR, et al. Race and comorbid factors predict nonalcoholic fatty liver disease histopathology in severely obese patients. Surg Obes Relat Dis 2005;1:6-11.

23. Garcia-Compean D, Jaquez-Quintana JO, Gonzalez-Gonzalez JA, et al. Liver cirrhosis and diabetes: risk factors, pathophysiology, clinical implications and management. World J Gastroenterol 2009;15:280-8.

24. Perseghin G, Mazzaferro V, Sereni LP, et al. Contribution of reduced insulin sensitivity and secretion to the pathogenesis of hepatogenous diabetes: effect of liver transplantation. Hepatology 2000;31:694-703.

25. Bonora E, Kiechl S, Willeit J, et al.; Bruneck study. Population-based incidence rates and risk factors for type 2 diabetes in white individuals: the Bruneck study. Diabetes 2004;53:1782-9.

26. Must A, Spadano J, Coakley EH, et al. The disease burden associated with overweight and obesity. JAMA 1999;282:1523-9.

27. Centers for Disease Control and Prevention (CDC). Incidence of end-stage renal disease among persons with diabetes-United States, 1990-2002. MMWR Morb Mortal Wkly Rep 2005;54:1097-100

28. Leske MC, Wu SY, Hennis A, et al.; Barbados Eye Study Group. Nine-year incidence of diabetic retinopathy in the Barbados Eye Studies. Arch Ophthalmol 2006; $124: 250-5$

29. Athyros VG, Mikhailidis DP, Didangelos TP, et al. Effect of multifactorial treatment on nonalcoholic fatty liver disease in metabolic syndrome: a randomised study. Curr Med Res Opin 2006;22:873-83.

Correspondence to: Dr. Gillian Booth, St. Michael's Hospital,

61 Queen St. E., 6th Floor, Toronto ON M5C 2T2;

boothg@smh.toronto.on.ca 